\title{
CROSSING REGIONS FOR SUPERSONIC CONES
}

\author{
R.T. Ferreyra ${ }^{1}$, J.P. Tamagno ${ }^{2}$
}

${ }^{1}$ Department of Mathematic, Faculty of Exact, Physical and Natural Sciences, National University of Córdoba, Argentina (ricardotf45@ hotmail.com)

${ }^{2}$ Department of Aeronautics, Faculty of Exact, Physical and Natural Sciences, National University of Córdoba, Argentina

\begin{abstract}
Recently, some new analytical methods have been developed and successfully applied to provide solutions for the Taylor-Maccoll boundary value problem. In this paper an analysis is carried out to justify the generation of crossing regions for lower cone angles. To this end, a key formulation between the free stream Mach number, the geometric cone angle and the shock wave properties, is provided.
\end{abstract}

Keywords: Shock wave, Cones, Compressible flow.

\section{INTRODUCTION}

The original study of a conical body of circular cross section at zero angle of attack inside a supersonic flow was developed by Taylor \& Maccoll 1933, see [1]. After this year a large amount of numerical and perturbation techniques were developed to approximate solutions and to deal with the problem.

In modern compressible flow literature, the same model and the hypotheses of stationary, non-rotational, constant entropy and null mass force and conic flow in spherical coordinates were developed with historical perspective by Anderson 2003, see [2]. However, the author claimed that there is no closed-form solution to the Taylor-Maccoll equation and that it must be solved numerically, and to expedite the numerical solution the author provided a numerical procedure by employing an inverse approach, that is the shock is assumed and the cone is calculated, in contrast with the direct strategy in which the cone is assumed and the shock is calculated.

Ferreyra \& Tamagno 2011 show that being $\alpha$ a real number and for lower cone angles (or lower $\theta_{C}$ values), the function $\alpha \cos \left(\theta-\theta_{C}\right)$ provides a good approximation for the boundary value problem, [3].

Although, this handy solution, called the Conic Solution, is not similar to existing solutions, the set of solutions obtained were demonstrated to be valid only for lower cone angles $20^{\circ}>\theta_{C}>0^{\circ}$, but inside the whole physical region $\theta_{S}^{\circ}>\theta>\theta_{C}^{\circ}$. 
Recent work by Ferreyra showed an analysis for higher cone angles, and as a result a closed form solution in the whole region $45^{\circ}>\theta_{C}>0^{\circ}$ was obtained, see [4].

In this paper, due to the fact that closed form solutions are now available, [3] and [4], and crossing region has been demonstrated to exist for lower cone angles, [3], an analytical explanation is provided for the crossing phenomena in agreement with the physical and geometrical intuition.

\section{CROSSING REGIONS FROM THE CONIC SOLUTION}

The dimensionless Taylor-Maccoll equation in the modern form, see [2], looks like as follows:

$$
\left(\frac{\gamma-1}{2}\right)\left(1-V_{R}^{2}-V_{\theta}^{2}\right)\left(2 V_{R}+V_{\theta} \cot \theta+V_{\theta \theta}\right)-V_{\theta}^{3}\left(V_{\theta \theta}+V_{R}\right)=0 .
$$

In Eq. (1), $\theta$ is an angle between the cone angle $\theta_{c}$ and the shock angle $\theta_{s}$, and, in spherical coordinates, the velocity vector components are indicated as $\left(V_{R}, V_{\theta}, V_{w}\right)$. The equation (1) has been obtained after the hypothesis of constant enthalpy. In addition, three boundary conditions are included to complete the physical model: $V_{\theta}\left(\theta_{C}\right)=0, V_{R}\left(\theta_{S}\right)=V_{1} \cos \left(\theta_{S}\right)$, and

$$
V_{\theta}\left(\theta_{S}\right)=-\frac{V_{1} \sin \left(\theta_{S}\right)}{\left(\frac{\gamma+1}{2}\right)}\left[\left(\frac{\gamma-1}{2}\right)+\frac{1}{M_{1}^{2} \sin ^{2}\left(\theta_{S}\right)}\right]
$$

where $V_{1}$ is the velocity of the current flow, $M_{1}$ is the mach number associated to $V_{1}$, and $\gamma$ is the ratio of specific heats.

Ferreyra \& Tamagno 2011 show that the Taylor-Maccoll boundary value problem is solved by the "Conic Solution"

$$
V_{R}(\theta)=V_{R}\left(\theta_{C}\right) \cos \left(\theta-\theta_{C}\right)
$$

in the region $20^{\circ}>\theta_{C}>0^{\circ}$. This solution allows to obtain crossing regions in $10^{\circ}>\theta_{C}>0^{\circ}$

\subsection{Crossing regions}

The constant value $V_{R}\left(\theta_{C}\right)$ is obtained by imposing $V_{R}\left(\theta_{S}\right)=V_{1} \cos \left(\theta_{S}\right)$ in Eq. (3). Also, the boundary condition $V_{\theta}\left(\theta_{C}\right)=0$ clearly follows from Eq. (3). Finally, the Conic Solution and all boundary conditions provide a relationship between $M_{1}, \theta_{S}$ and $\theta_{C}$. 


$$
\left|M_{1}\right|=\frac{1}{\left\lfloor\sin \left(\theta_{S}\right)\right\rfloor}\left\{\left(\frac{\gamma+1}{2}\right)\left(\frac{1}{\tan \left(\theta_{S}\right)}\right)\left|\frac{V_{\theta}\left(\theta_{S}\right)}{V_{R}\left(\theta_{S}\right)}\right| \operatorname{Sign}-\left(\frac{\gamma-1}{2}\right)\right\}^{-\frac{1}{2}} .
$$

Note that, for the Sign function there are two possible analytical descriptions

Table 1. The jump condition to cross regions (see [3])

\begin{tabular}{cc}
\hline Physical Condition & Sign \\
\hline$\theta_{C}=0^{\circ}$ & + \\
$20^{\circ}>\theta_{C}>0^{\circ}$ & - \\
\hline
\end{tabular}

Table 1 shows a correlation between the Sign function and the involved physics. In fact, since $\theta_{C}=0^{\circ}$ is the geometrical limit condition in which the cone begins to appear inside the flow, the tangential velocity must change the sign.

The curves $\theta_{C}^{*}=10^{\circ}, 7^{\circ}, 4^{\circ}$ and $2^{\circ}$ are crossed by the curve $\theta_{C}=0^{\circ}$ at $M \approx 1.95,1.7,1.4$ and 1.1 , respectively. Fig. 1 shows that $\theta_{C}^{*} \rightarrow \theta_{C}=0^{\circ} \Rightarrow M^{*} \rightarrow 1$.

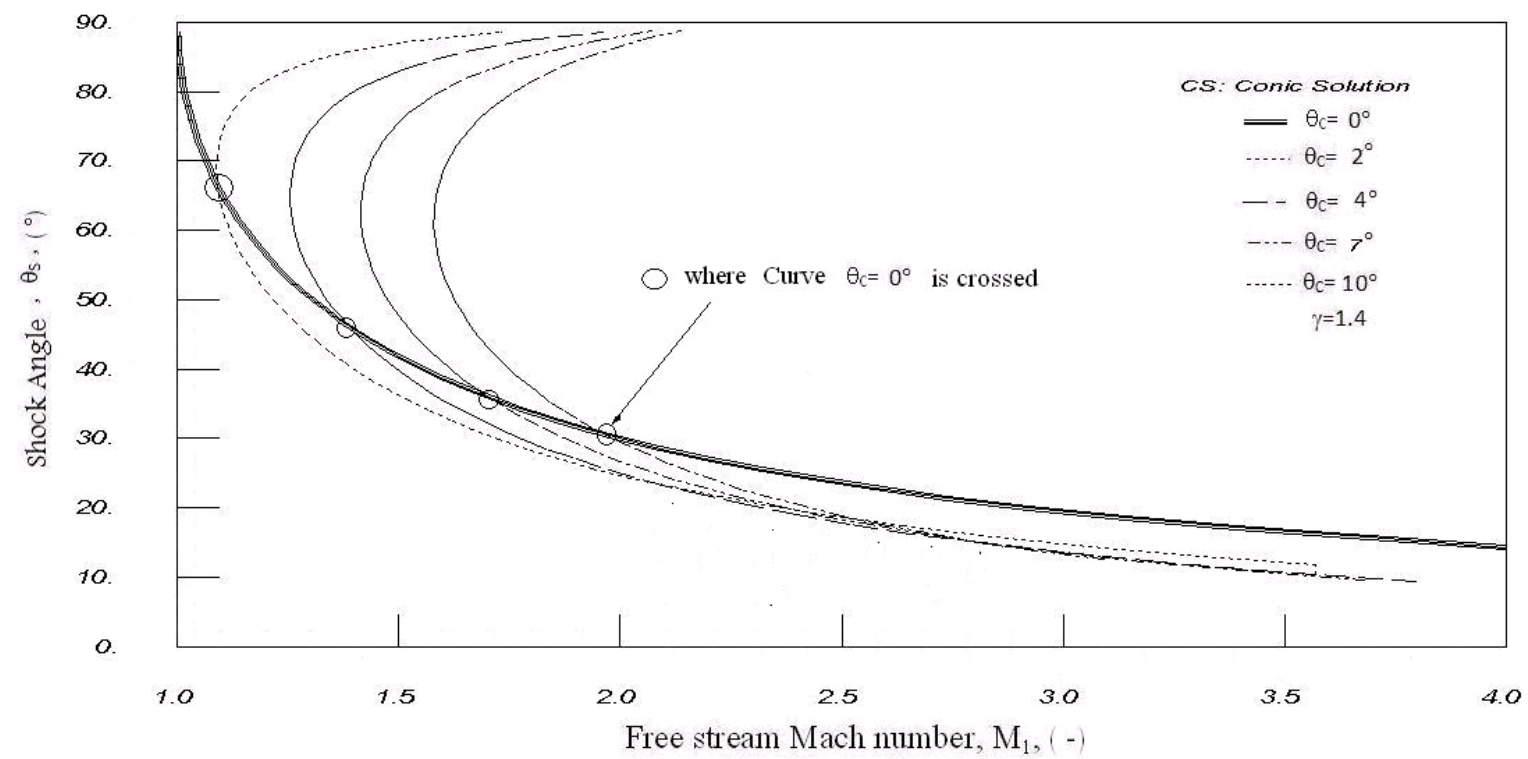

Figure 1. Shock angle and cone half angle vs. free stream Mach number, where $0^{\circ}<\theta_{C}<10^{\circ}$

To conclude, it has been shown from the Conic Solution that a crossing region appears under the condition $0^{\circ}<\theta_{C}<10^{\circ}$. 


\section{Acknowledgements}

Dr. Ricardo T. Ferreyra acknowledges the academic and research national program, PROMEI, Ministerio de Cultura y Educación de la República Argentina, for the financial support.

\section{REFERENCES}

[1] Taylor, G.I. \& Maccoll, J.W. 'The air pressure on a cone moving at high speeds', Proceedings of the Royal Society of London, Series A, London, vol. 139, pp. 278-29, 1933.

[2] Anderson, J.D., 'Modern Compressible Flow with Historical Perspective', Mc Graw Hill, Series in aeronautical and aerospace engineering, $3^{\text {rd }}$ edition, New York, pp. 1-375., 2003

[3] Ferreyra, RT \& Tamagno, J. P. 'A non local technique to approach the solutions of nonlinear boundary value problems applied to a supersonic flow model', International Federation of Nonlinear Analysis IFNA-ANS scientific Journal 'Problems of nonlinear Analysis in Engineering Systems’, 2012, (manuscript sent for Special Issue 2012).

[4] Ferreyra, R. T. 'New closed form solutions for Taylor and Maccoll Model', Journal of Fluid Mechanics, (sent for publication 2012), 2012. 